\title{
CICLO DE VIDA Y DESCRIPCIÓN MORFOLÓGICA DE HEILIPUS LAURI BOHEMAN (COLEOPTERA: CURCULIONIDAE) EN COLOMBIA
}

\section{LIFE CYCLE AND MORPHOLOGICAL DESCRIPTION OF HEILIPUS LAURI BOHEMAN (COLEOPTERA: CURCULIONIDAE) IN COLOMBIA}

\section{Valentina diaZ GRISALES, ${ }^{1, *}$ Ana Milena CAICEDO VALlEJO² y Arturo CARABALÍ MUÑoZ ${ }^{3}$}

\author{
${ }^{1}$ Ingeniera Agrónoma, Calle 48 No. 25-48 Piso 1, 170004, Manizales, Caldas, Colombia. \\ 2 Instituto Colombiano Agropecuario, Dirección Técnica de Epidemiología y Vigilancia Fitosanitaria Seccional \\ Antioquia, Kilómetro 7 Vía a Las Palmas, 054048, Rionegro, Antioquia, Colombia <anam.caicedo@gmail.com>. \\ ${ }^{3}$ Centro de Investigación Palmira, Corporación Colombiana de Investigación Agropecuaria Corpoica, Diagonal a \\ la intersección de la carrera 36A con calle 23, 763533, Palmira, Valle del Cauca, Colombia <acarabali@corpoica. \\ org.co>. \\ * Autor para correspondencia: <valentinadiazgrisales@gmail.com>.
}

Recibido: 01/08/2016; aceptado: 16/03/2017

Editor responsable: Carmen Huerta Crespo.

Diaz, V., Caicedo, A.M. y Carabalí, A. (2017). Ciclo de vida y descripción morfológica de Heilipus lauri Boheman (Coleoptera: Curculionidae) en Colombia. Acta Zoológica Mexicana (n.s.), 33(2), 231-242.

RESUMEN. Heilipus lauri Boheman (Coleoptera: Curculionidae) es una especie que afecta los frutos de Persea americana Miller (Lauraceae) y está catalogada como plaga cuarentenaria para el mercado de Estados Unidos. En Colombia se ha detectado en varios departamentos, no obstante, su manejo se dificulta debido a la escasa información sobre su biología y hábitos. Con el objetivo de contribuir al conocimiento de la biología de esta especie, se determinó el ciclo de vida en laboratorio $\left(21,6 \pm 1,8{ }^{\circ} \mathrm{C}\right.$ y humedad relativa de $\left.66 \pm 8 \%\right)$ y se realizó la descripción morfológica de cada uno de sus estados de desarrollo. El ciclo de vida de huevo hasta la emergencia del adulto duró 76,14 \pm 7,31 días. De éstos, la etapa de huevo tuvo una duración de 12,70 \pm 1,87 días, el estado larval 48,68 \pm 6,41 días y la pupa 14,76 $\pm 1,13$ días. Estos resultados representan el primer registro de duración del ciclo de vida de especímenes de $H$. lauri asociados a P. americana cv. Hass en Colombia, y podrían constituir la base para el desarrollo e implementación de programas de manejo integrado de plagas.

Palabras clave: Persea americana, Cultivar Hass, Plaga cuarentenaria, Biología, Cría, Barrenador del hueso del aguacate.

\section{INTRODUCCIÓN}

El aguacate (Persea americana Miller) (Lauraceae) es una fruta tropical con una demanda creciente en los mercados internos y externos, gracias a su aceptado consumo en fresco, sus cualidades nutracéuticas y múltiples usos, tanto en la industria alimenticia como en la cosmética y farmacéutica (ICA, 2012; Peña, 2013; Sandoval et al.,
Diaz, V., Caicedo, A.M., \& Carabalí, A. (2017). Life cycle and morphological description of Heilipus lauri Boheman (Coleoptera: Curculionidae) in Colombia. Acta Zoológica Mexicana (n.s.), 33(2), 231-242.

ABSTRACT. Heilipus lauri Boheman is a species that affects the fruits of Persea americana Miller (Lauraceae) and is catalogued as a quarantine pest for United States market. In Colombia, it has been detected in several departments, however, its management is difficult because of the limited information about its biology and habits. With the aim of contributing to the knowledge of the biology of this species, the life cycle under laboratory conditions $\left(21.6 \pm 1.8^{\circ} \mathrm{C}\right.$ and relative humidity of $66 \pm 8 \%$ ) was determined and morphological description of the developmental stages for the species was carried out. The life cycle ranging from egg to adult took lasted $76.14 \pm 7.31$ days in average. Of these, the egg stage lasted $12.70 \pm 1.87$ days, the larval stage encompassed $48.68 \pm 6.41$ days and the pupa $14.76 \pm 1.13$ days. These results represent the first record of duration of the life cycle of specimens of $H$. lauri associated with P. americana cv. Hass in Colombia, and might constitute the base for the development and implementation of integrated pest management.

Key words: Persea americana, Hass cultivar, Quarantine pest, Biology, Rearing, Avocado seed borer.

2014). En Colombia, el área sembrada de aguacate presentó un incremento sustancial durante la última década, en respuesta al aumento del consumo per cápita y a su potencial exportador como fruta fresca y procesada (Ramírez et al., 2014). Esta oportunidad de exportación ha impulsado el establecimiento de nuevas áreas, especialmente del cultivar 'Hass', el cual se destaca por ser el más comercializado en el mundo. Sin embargo, existen 
en la actualidad restricciones de tipo fitosanitario que impiden su entrada a otros mercados (Peterson \& Orden, 2008; ICA, 2016), atribuidas a la presencia de insectos plaga en el país pero que no se encuentran en los países de destino como Estados Unidos, uno de los principales consumidores e importadores de aguacate en el mundo (Carabalí et al., 2009). Tal es el caso de los curculiónidos Heilipus lauri (Boheman, 1845) y Heilipus trifasciatus (Fabricius, 1787) (Coleoptera: Curculionidae), y el lepidóptero Stenoma catenifer (Walsingham, 1912) (Lepidoptera: Oecophoridae), especies recientemente declaradas por el Instituto Colombiano Agropecuario (ICA) plagas de control oficial, pues generan un impacto económico negativo en la producción de $P$. americana y limitan el acceso de frutos frescos a nuevos mercados (ICA, 2016).

Heilipus lauri es una especie que afecta frutos criollos y variedades mejoradas de $P$. americana y está catalogada como plaga cuarentenaria para el mercado de Estados Unidos (Castañeda-Vildózola et al., 2012; ICA, 2016). Además del ataque a frutos, los adultos causan daño en el follaje y brotes jóvenes; las hembras ovipositan en el fruto y la larva se alimenta de la semilla, ocasionando su destrucción total o parcial y la caída prematura del futo (Hoyos \& Giraldo, 1984). En México, Medina et al. (2010) concluyeron que en huertas comerciales de Tepoztlán, Morelos, este barrenador tuvo mayor preferencia por frutos del cultivar 'Hass', reportando daño en el 59,5\% de los frutos muestreados.

Boheman (1845), describió a H. lauri a partir de especímenes recolectados en México; posteriormente, Champion (1902) menciona esta especie en Calpulalpan, Tlaxcala, México, en su obra Biologia Centrali Americana. Barber (1919) señaló las semillas de Persea persea de México como su hábitat. Castañeda (2008), documentó que en este país $H$. lauri está diseminado en la provincia del eje volcánico transmexicano siguiendo la distribución natural de Persea americana var. drymifolia (Lauraceae) y los cultivares comerciales de aguacate Hass, Fuerte, Colín V-33 y otros a altitudes de 1290 a 1920 msnm. Su presencia también ha sido registrada en Costa Rica, Guatemala, Nicaragua y Honduras (Hoyos \& Giraldo, 1984; Rubio et al., 2009; EPPO, 2016; Palacios-Torres et al., 2016).

Wibmer \& O'Brien (1986), no mencionan la presencia de $H$. lauri en América del sur; sin embargo, Gallego (1949), Hoyos \& Giraldo (1984), Caicedo et al. (2010) y Carabalí (2014), reportaron su presencia en Colombia, donde se ha detectado en los departamentos de Antioquia,
Caldas, Cauca, Quindío, Risaralda, Tolima y Valle del Cauca (Hoyos \& Giraldo, 1984; Rubio et al., 2009; ICA, 2015). En núcleos productores de aguacate cv. Hass del oriente de Antioquia y el norte del Tolima, la incidencia de $H$. lauri fue superior en comparación con $S$. catenifer, según resultados de la vigilancia sistemática del ICA durante 12 meses. En el núcleo productor del oriente Antioqueño se reportó la presencia de $H$. lauri en $14,4 \%$ de los predios monitoreados, mientras que $S$. catenifer se registró en 6,1\% de éstos. En relación con el núcleo productor del norte del Tolima, 25\% de los predios evaluados resultaron positivos a $H$. lauri y $2,5 \%$ a $S$. catenifer (ICA, 2014).

Aspectos biológicos de esta especie previamente se estudiaron en México por García (1962). El trabajo incluye la duración del ciclo de vida y la descripción de los hábitos del insecto, así como un estudio morfológico detallado de cada uno de sus estados de desarrollo; sin embargo, no especifica las variables de temperatura, humedad relativa $\mathrm{y}$ fotoperiodo empleadas en el trabajo de laboratorio. Posteriormente, Castañeda (2008) determinó el ciclo biológico, número de estadios larvarios, fertilidad, fecundidad y longevidad de esta especie bajo condiciones controladas; además, realizó una descripción morfológica del huevo, larva de IV estadio, pupa y adulto, incluyendo la genitalia masculina y femenina. En Colombia, el único estudio publicado sobre biología de $H$. lauri es el realizado por Caicedo et al. (2010), quienes caracterizaron el daño en el fruto y reportaron una duración de 15,14 \pm 0,33 días para el estado de pupa.

El desconocimiento de la bioecología de esta especie en las condiciones locales dificulta su control y ha llevado a los agricultores a realizar aplicaciones indiscriminadas de insecticidas para su manejo en campo; estas medidas son contrarias a las exigencias de los mercados internacionales en relación con los bajos niveles de residuos de plaguicidas en productos frescos (Manrique et al., 2014). Con el objetivo de contribuir al conocimiento de la biología de $H$. lauri en Colombia, se determinó el ciclo de vida bajo condiciones de laboratorio y se realizó la descripción morfológica de cada uno de sus estados de desarrollo, aspectos fundamentales para diseñar estrategias de manejo integrado de esta especie plaga.

\section{MATERIALES Y MÉTODOS}

Localización. El estudio se realizó de enero a julio de 2014, en el laboratorio de entomología del Centro de In- 
vestigación La Selva $\left(21,6 \pm 1,8{ }^{\circ} \mathrm{C}\right.$ y humedad relativa $66 \pm 8 \%$ ) de la Corporación Colombiana de Investigación Agropecuaria Corpoica, ubicado en el municipio de Rionegro, Antioquia (6º7'46.4' ' LN, 75²4'51,8' ' LW), a una altitud de $2120 \mathrm{msnm}$.

Material vegetal e insectos. El establecimiento del pie de cría se realizó con frutos y semillas afectadas por $H$. lauri, procedentes de cultivos comerciales de $P$. americana cv. Hass ubicados en los municipios de Marinilla y El Retiro (Antioquia, Colombia). Los frutos y semillas utilizados para alimentar adultos y larvas provenían de cultivos comerciales de aguacate cv. Hass sin aplicaciones de insecticidas. El ciclo biológico se desarrolló con la progenie de los insectos criados en laboratorio.

Cría de Heilipus lauri. Los adultos obtenidos a partir de las semillas afectadas provenientes de campo se confinaron en cajas plásticas con tapa (40 cm x $30 \mathrm{~cm}$ x $32 \mathrm{~cm}$ ) y papel toalla en el fondo; para favorecer la aireación al interior del recipiente, se recortó un rectángulo $(20,5 \mathrm{~cm}$ x $26 \mathrm{~cm}$ ) de la tapa y se reemplazó por muselina blanca. Como fuente de alimentación y sustrato de oviposición se colocaron frutos de aguacate cv. Hass de aproximadamente 8 a 9 meses de edad, los cuales se renovaron cada dos días. Diariamente se revisaron los frutos para extraer los huevos, realizando cortes en los orificios de oviposición con ayuda de un bisturí metálico de punta de lanza. Los huevos se extrajeron de los frutos con un pincel de pelo de marta No. 000 y se individualizaron en recipientes plásticos esterilizados ( $2 \mathrm{~cm} \times 2 \mathrm{~cm}$ ), con tapa y toalla de papel en la base como sustrato de incubación, humedecida diariamente con agua destilada estéril. Las larvas neonatas se introdujeron en las semillas de aguacate cv. Hass desprovistas de la cubierta seminal, partidas por la mitad y con una pequeña perforación realizada con el bisturí; cada larva se introdujo en el orificio utilizando un pincel con las características previamente mencionadas y se cubrieron con residuos de la perforación. El conjunto larva-semilla fue dispuesto en recipientes plásticos esterilizados (11,2 cm x 9,2 cm x 7,4 cm), perforados en la parte superior y con una capa de aserrín esterilizado de un $1,0 \mathrm{~cm}$ de espesor en el fondo; el aserrín fue humedecido cada tres días con agua destilada estéril hasta la emergencia de adultos de las semillas.

Ciclo de vida. Un total de 300 adultos $\left(150 q, 150{ }^{\Uparrow}\right)$ obtenidos de la cría en laboratorio se confinaron en cámaras de oviposición, construidas con seis recipientes plásticos con tapa (40 cm x $30 \mathrm{~cm}$ x $32 \mathrm{~cm}$ ). Cada cámara de oviposición estaba constituida por 25 parejas y siete frutos de aguacate cv. Hass como fuente de alimentación y sus- trato de oviposición. Transcurridas 24 horas se retiraron los frutos, de su interior se extrajeron 253 huevos que fueron individualizados en recipientes plásticos $(2 \mathrm{~cm} \times 2$ $\mathrm{cm}$ ) con tapa y papel toalla en el fondo como sustrato de incubación. Los recipientes se marcaron con un número que identificó al individuo durante todo el estudio y se revisaron diariamente para humedecer la toalla con agua destilada estéril. Se registró la emergencia de cada larva, determinando así el periodo de incubación del huevo. Las larvas recién emergidas se utilizaron en la infestación artificial (manual) de semillas de P. americana cv. Hass; en esta etapa se emplearon 104 larvas neonatas y cada una se introdujo en media semilla. El conjunto larva-semilla se dispuso en un recipiente plástico perforado, rotulado con el número de la muestra y con aserrín esterilizado húmedo en la base. Transcurridos 35 días desde la infestación, a cada semilla se le realizó un orificio para determinar la supervivencia de las larvas introducidas; las semillas con larvas vivas en su interior se revisaron diariamente a través del orificio, con el fin de registrar el evento de pupación y definir el número de días requerido para completar el estado larval. De manera similar, mediante observación diaria de las pupas a través del orificio se cuantificó la duración de este estado de desarrollo, el cual finalizó al detectar la presencia de adultos al interior de las semillas. Se registró la relación de sexos en 97 individuos adultos emergidos de semillas infestadas artificialmente.

Durante el tiempo de evaluación se llevaron registros diarios de temperatura y humedad relativa del laboratorio. Los datos obtenidos en el ciclo de vida se agruparon en valores mínimos, máximos y promedios, acompañados de la desviación estándar.

Descripción morfológica. Se describieron las principales características morfológicas externas de los estados de desarrollo de $H$. lauri, visibles bajo un microscopio estereoscópico (Nikon SMZ 745T). Para las mediciones de cada uno de los estados biológicos se tomaron fotografías con una cámara Nikon DS-Fi2 adaptada a un estéreo microscopio Nikon SMZ 1500; las imágenes digitalizadas se midieron con la ayuda de software para microscopía (NIS-Elements D versión 4.0), utilizando milímetros como unidad de medida. El número de muestras empleadas y la terminología usada en cada caso se describen a continuación.

Huevo. Se utilizó una muestra de 30 huevos y se determinó el ancho y el largo, tomando como referencia los puntos más externos de sus bordes. La descripción se realizó según la terminología propuesta por García (1962) y Castañeda (2008). 
Larva. Para realizar la descripción de la larva madura así como de la larva de primer estadio, se tomó una muestra de 30 individuos de cada estadio. Se registró la longitud y el ancho de la cápsula cefálica (distancia entre las genas) y se consideraron los caracteres propuestos por García (1962), May (1993), Marvaldi (2003) y Vallejo et al., (2007).

Pupa. En una muestra de 30 pupas se determinó la longitud, tomada desde el margen anterior del pronoto hasta el margen posterior del abdomen, y se usaron los caracteres morfológicos y terminología propuesta por García (1962), May (1981), Marvaldi \& Morrone (1998) y Vallejo et al., (2007).

Adulto. Se utilizaron 30 adultos $(15 \circ, 15 \AA)$ y de cada uno se tomaron las siguientes medidas: longitud del cuerpo excluyendo el rostro, longitud del rostro, largo y ancho del pronoto, largo y ancho de los élitros. La descripción de las partes del cuerpo se realizó empleando la terminología propuesta por Costa Lima (1956), García (1962), Craw (1999), Sousa et al. (2004), Marvaldi \& Lanteri (2005), Vanin \& Gaiger (2005) y Vallejo et al., (2007).

\section{RESULTADOS Y DISCUSIÓN}

Hábitos. La biología de H. lauri está estrechamente ligada a la disponibilidad de frutos de aguacate. La hembra realiza horadaciones circulares sobre el fruto, posteriormente deposita el huevo en la perforación y lo empuja con su rostro hacia el fondo, para ubicarlo cerca de la semilla (Fig. 1A). Los orificios donde ocurrió la oviposición son cubiertos con los residuos derivados de la perforación. Las larvas emergentes barrenan a través de la pulpa hasta encontrar la semilla, se introducen y se alimentan sin llegar a destruirla (Fig. 1B). Las larvas presentan canibalismo, debido a esto suele encontrarse un solo individuo en cada semilla, aunque también es frecuente observar dos larvas, una por cotiledón. Próxima a la pupación, la larva tiene poca movilidad y convierte el interior de la semilla consumida en una cámara de pupación donde se aloja. El estado de pupa ocurre en el interior de la semilla (Fig. 1C). Finalmente, el adulto realiza un orificio circular con sus mandíbulas para abandonarla (Figs. 1 D-E). Los adultos se alimentan de hojas, brotes, tallos tiernos y frutos; es frecuente observarlos copulando sobre el fruto.
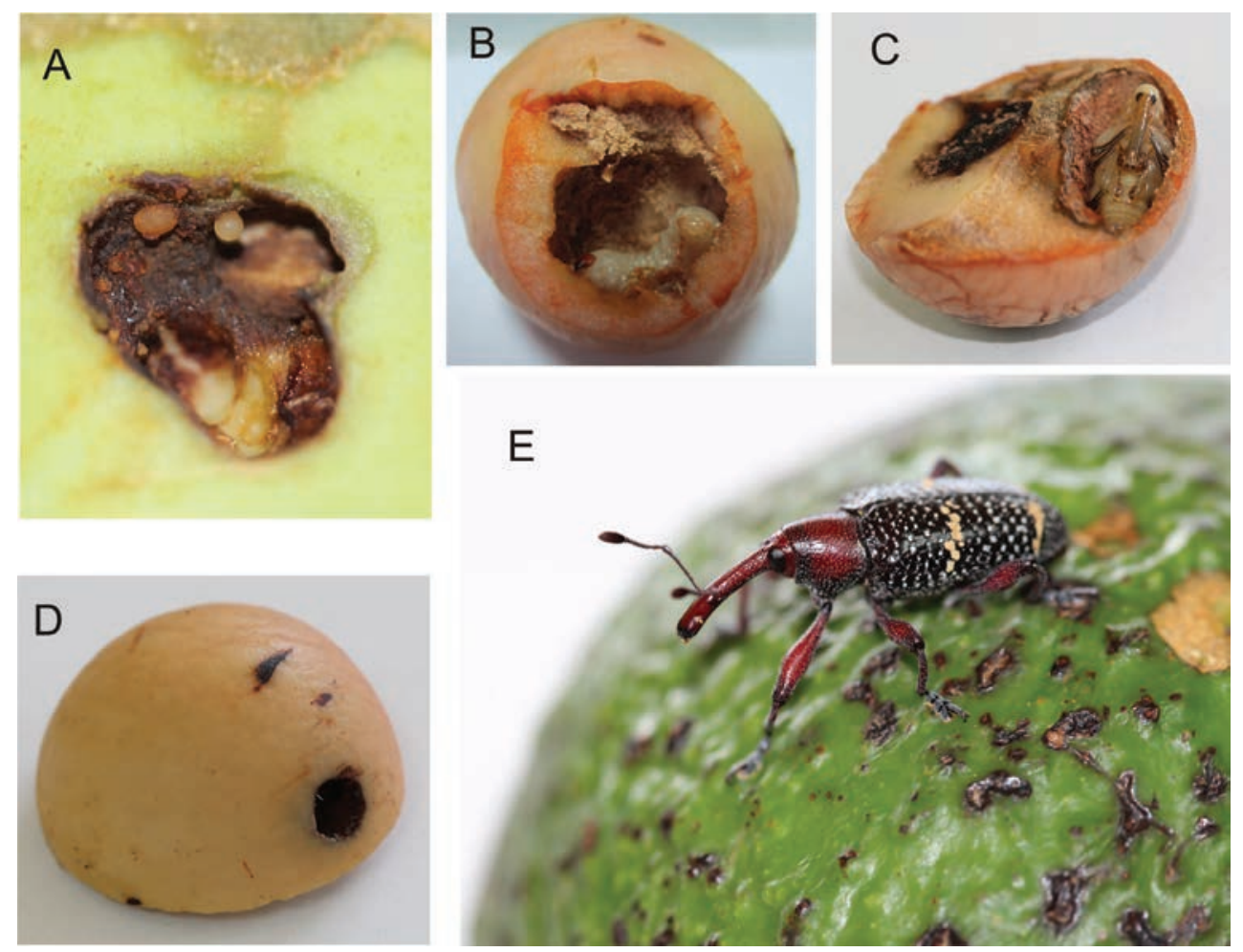

Figura 1. Ciclo de vida de Heilipus lauri: A. Huevos al interior del fruto B. Larva consumiendo semilla C. Pupa en semilla D. Semilla con orificio realizado por el adulto para emerger E. Adulto. 


\section{Ciclo de vida}

Huevo. El periodo de incubación del huevo fue de 12,70 $\pm 1,87$ días $(\mathrm{n}=50)$. Este resultado es cercano al período de incubación de 12,96 días reportado por García (1962), aunque el trabajo no especifica las condiciones de temperatura, humedad relativa y fotoperiodo. Castañeda (2008) señaló una duración de 10,87 \pm 0,45 días para la incubación del huevo de $H$. lauri en laboratorio (26 \pm 2 ${ }^{\circ} \mathrm{C}$, humedad relativa de 60 - 70\% y fotoperiodo 12:12), resultado que se encuentra dos días por debajo del obtenido en el presente estudio y podría explicarse por la diferencia en las condiciones ambientales. El período de incubación registró valores mínimos y máximos de 11 y 17 días respectivamente, con coeficiente de variación de 14,69\%; este intervalo es más amplio que el reportado por García (1962) de 12 a 15 días, y por Castañeda (2008) de 10 a 13 días.

Viabilidad del huevo. De una cohorte de 253 huevos emergieron 211 larvas, lo cual representa una viabilidad de 83,40\%. Castañeda-Vildózola et al. (2012) reportaron una fertilidad de $85,57 \pm 7,27 \%$, cuantificada a partir del número de larvas que emergieron de una muestra total de 1646 huevos recolectados durante tres periodos. En $S$. catenifer, otra especie catalogada como plaga cuarentenaria para el mercado de Estados Unidos y de importancia económica en cultivos de aguacate cv. Hass en Colombia, la viabilidad de la fase de huevo en laboratorio (25 \pm 1 ${ }^{\circ} \mathrm{C}$, humedad relativa de $70 \pm 10 \%$ y fotoperiodo de $14 \mathrm{~h}$ ) fue superior a la registrada en el presente estudio para $H$. lauri, alcanzando un $94,44 \%$ (Silva et al., 2006).

Larva. El estado larval tuvo una duración de 48,68 \pm 6,41 días ( $\mathrm{n}=50$ ), lo cual coincide con lo registrado por Castañeda (2008), quien concluyó que las larvas de $H$. lauri alimentadas con frutos de aguacate cv. Colín V-33 completan su desarrollo en 48,51 \pm 2,30 días, pasando por cuatro estadios larvales. Por el contrario, en condiciones ambientales no controladas García (1962) reportó una duración del estado de larva de 58,58 días y cinco probables estadios larvarios, cada uno con una duración promedio de 10,8 a 12,6 días. En el presente estudio, la duración del estado larval presentó un rango de variación entre 39 y 65 días, siendo mayor en comparación con el reportado por García (1962) y Castañeda (2008), quienes registraron rangos de 54 - 63 días y 44 - 55 días, respectivamente.

El desarrollo larvario está influenciado tanto por las condiciones ambientales como por las características del recurso alimenticio, por lo tanto, aspectos como la calidad y cantidad de semilla suministrada a la larva tienen efecto sobre las características del adulto. Stearns (1992) citado por Nylin \& Gotthard (1998), advierte que un efecto comúnmente observado en respuesta a la reducción de los recursos alimenticios es una tasa de crecimiento más baja, lo cual resulta en un tiempo de desarrollo prolongado y un tamaño reducido en la etapa adulta. En este sentido, observaciones realizadas a la cría del insecto en laboratorio sugieren que semillas pequeñas dan origen a individuos de un tamaño más reducido en comparación con aquellos adultos que en su estado larval son alimentados con semillas más grandes, aun cuando todas provengan de frutos del cultivar Hass.

Pupa. El estado pupal registró una duración de 14,76 \pm 1,13 días $(n=50)$ con variaciones entre 13 y 17 días. Este resultado es similar al obtenido por García (1962) de 15,53 días con un rango de 14 - 16 días, y por Castañeda (2008) de 15,32 \pm 1,58 días con un rango de 11 - 18 días. Caicedo et al. (2010) determinaron el tiempo de desarrollo del estado pupal en condiciones de casa de malla (26,1 $\pm 0,3{ }^{\circ} \mathrm{C}$ y humedad relativa de $71 \%$ ) con material colectado en campo, sin establecer la cría del insecto, reportando una duración de 15,14 \pm 0,33 días con un rango de 11 - 18 días. Con un coeficiente de variación de 7,69\%, los valores de la duración del estado de pupa fueron los que presentaron una menor variabilidad.

Ciclo de huevo a adulto. En laboratorio, y utilizando semillas de aguacate cv. Hass como fuente de alimentación de las larvas, el tiempo total del ciclo desde huevo hasta la emergencia del adulto fue de 76,14 \pm 7,31 días con un rango de 67 - 98 días. Castañeda (2008) menciona una duración de 74,68 \pm 1,71 días con variaciones entre 72 y 80 días, en tanto que Salgado \& Bautista (1993) indican que H. lauri requiere 100 a 115 días para su desarrollo desde huevo hasta la emergencia del adulto en Ixtapan de la Sal, Estado de México, aunque no determinaron la duración de cada uno de los estados biológicos.

Relación de sexos. Se encontró una proporción de hembras y machos de 1: 0,9, correspondiente a 51 hembras: 46 machos. Este resultado es igual al reportado por Castañeda (2008).

\section{Descripción morfológica}

Huevo. El huevo de $H$. lauri mide 1,26 $\pm 0,10 \mathrm{~mm}$ de largo ( $\mathrm{n}=30$; rango $1,09-1,50 \mathrm{~mm})$ y $0,84 \pm 0,04 \mathrm{~mm}$ de ancho ( $\mathrm{n}=30$; rango $0,72-0,93 \mathrm{~mm}$ ), es ovalado, presenta el corión reticulado formando figuras pentagonales y hexagonales (Fig. 2A). Tras la oviposición, el huevo es 

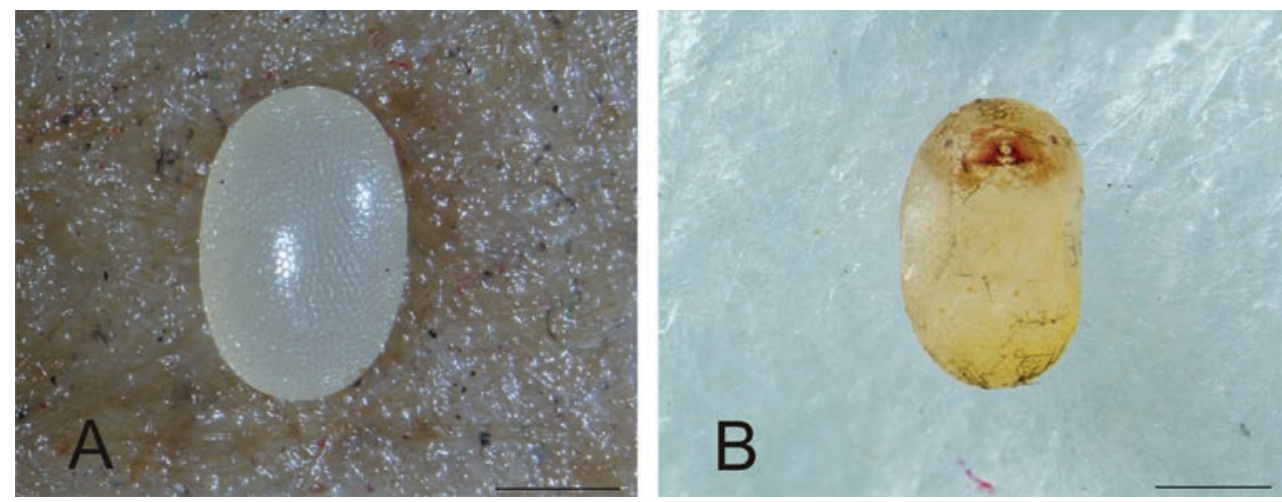

Figura 2. Heilipus lauri, huevo: A. Huevo recién puesto B. Larva próxima a emerger. Escala: 0,5 mm.

de color blanco opaco y a medida que avanza el desarrollo embrionario se torna marrón claro. Una vez la larva está próxima a emerger, se observa su cápsula cefálica a través del corión (Fig. 2B).

Larva I. La larva de primer estadio mide 1,91 \pm 0,31 mm de largo ( $\mathrm{n}=30$; rango $1,22-2,35 \mathrm{~mm}$ ), la cápsula cefálica mide 0,54 $\pm 0,03 \mathrm{~mm}$ de ancho $(\mathrm{n}=30$; rango $0,44-0,59$ $\mathrm{mm}$ ) (Figs. $3 \mathrm{~A}-\mathrm{B}$ ). La larva neonata es similar a la larva madura en todos los caracteres taxonómicos, excepto en la coloración del tórax, el cual es hialino; en el pronoto las dos manchas amarillas apenas son visibles.

Larva madura. La larva de último estadio mide 19,91 $\pm 1,42 \mathrm{~mm}$ de largo ( $\mathrm{n}=30$; rango $16,90-22,20 \mathrm{~mm}$ ), es curvada, robusta, ápoda y el cuerpo es de color blanco opaco. Presenta setas cortas, delgadas y de color marrón claro, tanto en la cabeza como en el tórax y el abdomen. La cápsula cefálica bien desarrollada y esclerotizada, mide 2,01 \pm 0,12 $\mathrm{mm}$ de ancho ( $\mathrm{n}=30$; rango 1,85 - 2,27 $\mathrm{mm}$ ), de color marrón claro y forma ovalada, tipo hipognata. Presenta setas cortas, delgadas, algunas un poco más largas que otras. Línea ecdisial en forma de "Y" invertida, visible en toda su longitud (Fig. 4A). Antenas de un segmento, sensorio antenal cónico. Aparato bucal de tipo masticador, con mandíbulas de color negro fuertemente esclerosadas y bidentadas en el ápice. Las tormas labrales unidas en la base (Fig. 4B).

Tórax de color blanco cremoso, más claro que el abdomen. Protórax con un par de espiráculos laterales bíforos de color marrón claro (Fig. 4C). El pronoto no está dividido, presenta tres pares de setas y dos manchas débilmente esclerosadas de color amarillo oscuro, una a cada lado de la línea media. Mesonoto y metanoto divididos en dos pliegues: prodorso y postdorso (Fig. 4D); ambos segmentos torácicos con dos pares de setas postdorsales cortas y delgadas.

Abdomen de color blanco opaco, formado por diez segmentos. Los segmentos I a VII presentan un par de espiráculos bicamerales de color marrón claro ubicados lateralmente, el segmento VIII con espiráculos en posición dorsal (Figs. 4 E-F) y los segmentos IX y X sin es-
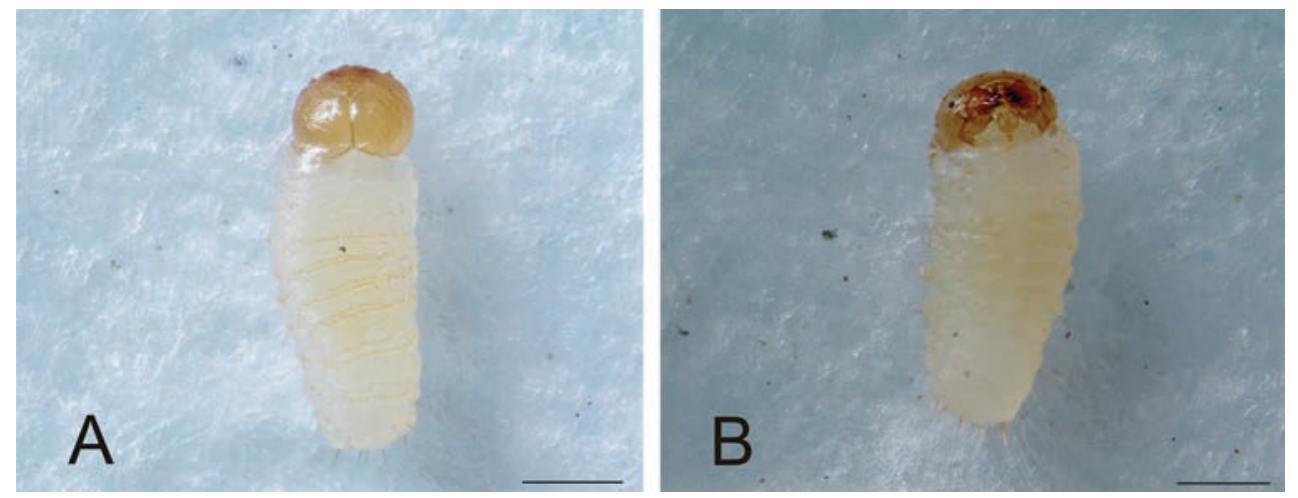

Figura 3. Heilipus lauri, larva de primer estadio: A. Vista dorsal B. Vista ventral. Escala: 0,5 mm. 

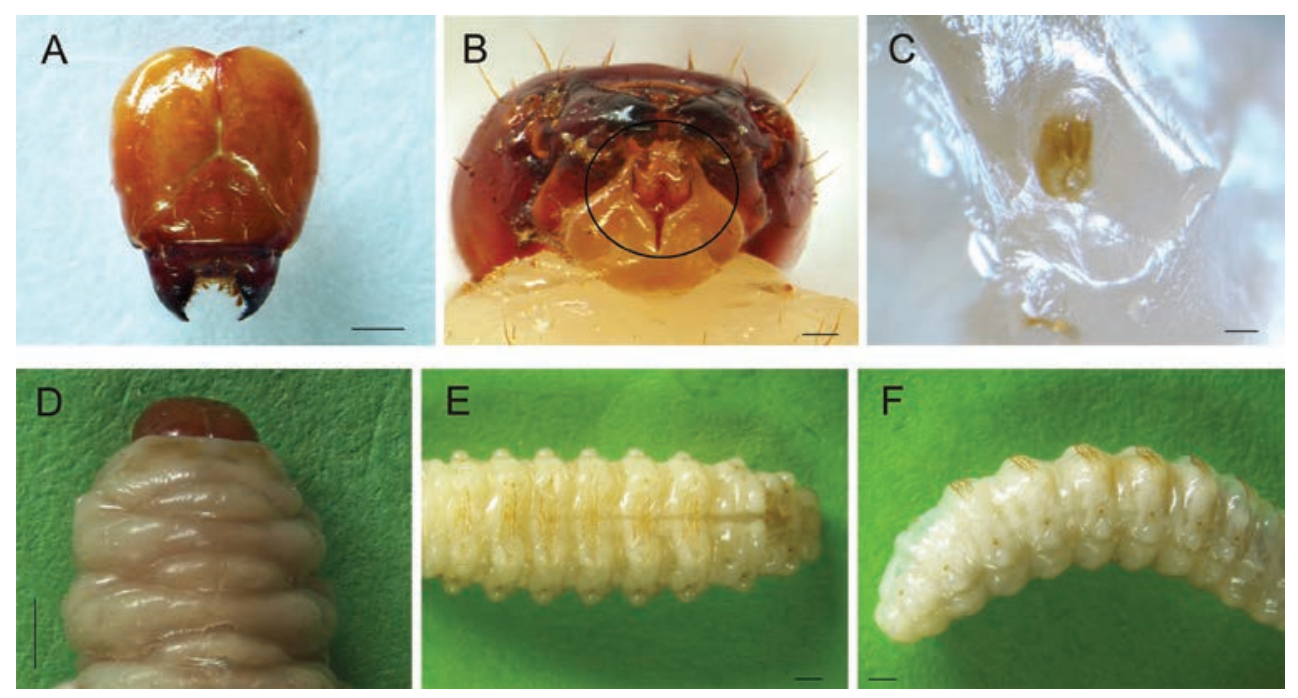

Figura 4. Heilipus lauri, larva madura: A. Cápsula cefálica, vista dorsal B. Cápsula cefálica, vista ventral. Nótese las tormas labrales (círculo negro) C. Espiráculo protorácico D. Tórax, vista dorsal. Nótese las dos manchas oscuras en el pronoto E. Abdomen, vista dorsal F. Abdomen, vista lateral. Escalas: A: 0,5 mm; B: $1 \mathrm{~mm}$; C: 0,1 mm; D-F: $1 \mathrm{~mm}$.

piráculos. Segmentos II a VII divididos transversalmente en cuatro pliegues dorsales, segmentos I y VIII cada uno con tres pliegues dorsales; los segmentos IX y X no están divididos. En los segmentos I a VII se observan seis pares de setas: dos pares en el postdorso; uno por encima del espiráculo; un par de setas en la epipleura; dos pares en la pleura, una larga y otra diminuta. El segmento VIII con un par de setas postdorsales, las setas en pleura y epipleura iguales que en los segmentos I a VII. El segmento IX está formado por cuatro lóbulos: uno dorsal que exhibe un par de setas; dos laterales, cada uno con una seta; uno ventral. Ano rodeado por cuatro lóbulos correspondientes al segmento $X$, visible ventralmente.

Pupa. Mide 16,03 \pm 1,64 mm de largo ( $\mathrm{n}=30$; rango 12,90 - 21,10 mm), tipo exarata, adéctica, de forma ovalada, color blanco cremoso y textura suave. En la superficie se observan setas cortas, gruesas y de color marrón oscuro. Cápsula cefálica en posición ventral, cubre el protórax. En la cabeza y el pico se distribuyen ocho pares de setas cortas y delgadas. Los ojos son ovalados y del mismo color del cuerpo cuando la pupa está recién transformada (Fig. $5 \mathrm{~A}$ ), gradualmente adquieren una coloración negra. Antenas visibles, hialinas; se distingue el escapo, el funículo y la maza. En el macho, el rostro alcanza el tarsómero III de las patas protorácicas, en la hembra se prolonga hasta el último tarsómero de las mesotorácicas. En el extremo del rostro, las mandíbulas comienzan a esclerosarse a medida que la pupa madura.
Protórax y metatórax similares en tamaño, mesotórax más pequeño (Fig. 5B). El protórax tiene forma subtriangular y bordes redondeados; presenta un par de espiráculos laterales de color marrón claro, cerca del límite con el mesotórax. Pronoto con nueve pares de setas largas, gruesas, de color marrón oscuro (Fig. 5C); mesonoto y metanoto con dos pares de setas cortas y delgadas. Hacia los lados del mesonoto se proyectan las pterotecas, hialinas y con estrías paralelas a sus bordes. En la pupa recién formada las patas también son hialinas; los fémures exhiben un par de setas en el ápice. En los tarsos, las uñas son las primeras en quitinizarse.

En el abdomen, los segmentos I-VII visibles dorsalmente (Fig. 5D); los segmentos VIII y IX curvados hacia la cara ventral (Fig. 5E). Segmentos I a VII cada uno con cuatro pares de setas dorsales; segmento VIII con un par de setas en el dorso. Los segmentos I a V presentan un par de espiráculos laterales (Figs. 5 F-G), los demás segmentos sin espiráculos. En vista ventral, el segmento IX muestra un par de proyecciones cónicas orientadas caudalmente, cada una con una seta en la base (Fig. 5H).

Adulto. El integumento es opaco; cabeza, rostro, pronoto y fémures de color rojo oscuro; las demás partes del cuerpo de color negro. Cuerpo de forma subromboidal, provisto de escamas filiformes variables en grosor y longitud, que se insertan en perforaciones generalmente pequeñas y de distinta profundidad. La longitud del cuerpo tomada desde la margen anterior del protórax hasta el ápi- 

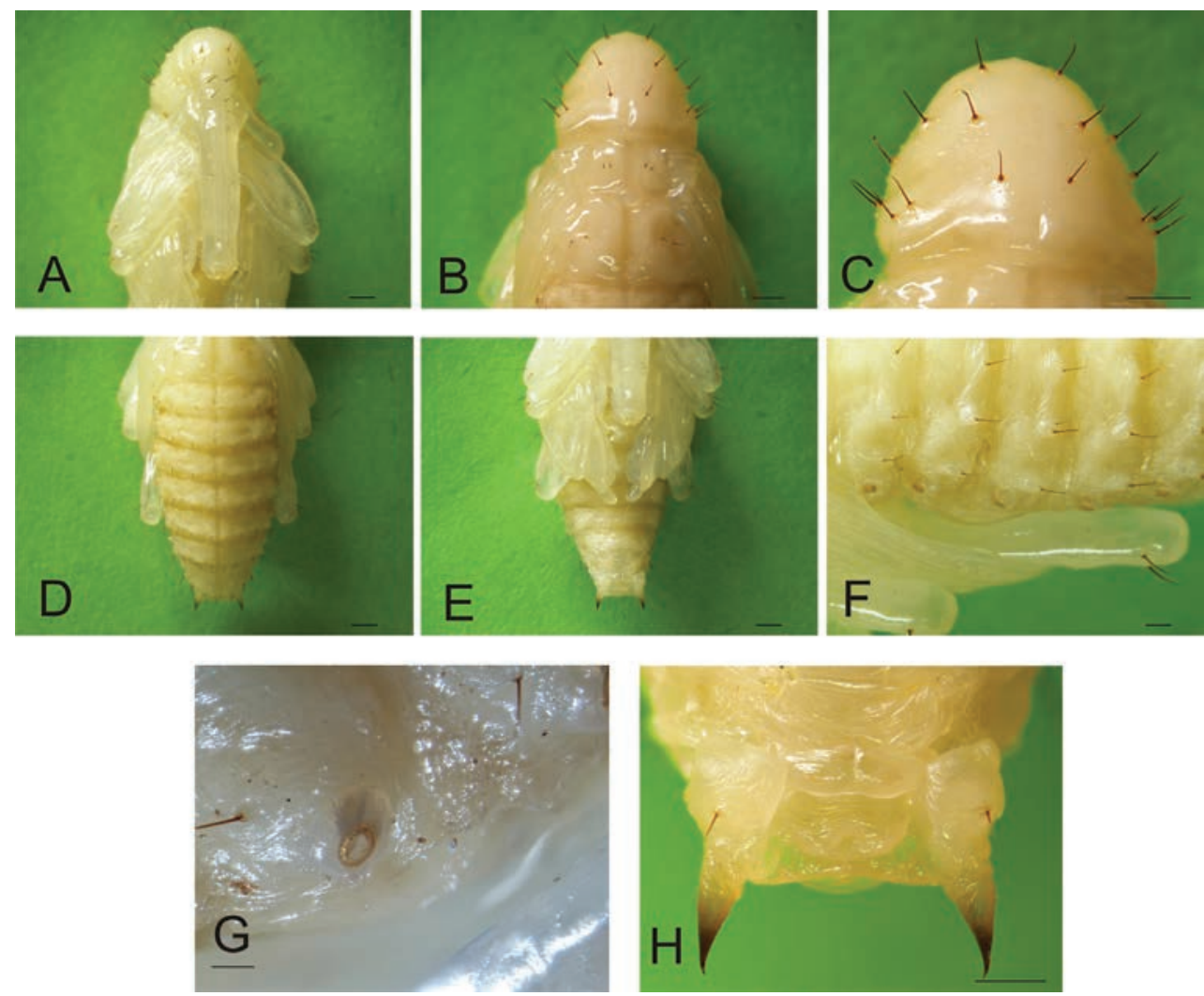

Figura 5. Heilipus lauri, pupa recién transformada A. Cabeza y pico, vista ventral B. Segmentos torácicos, vista dorsal C. Pronoto, vista dorsal D. Abdomen, vista dorsal E. Abdomen, vista ventral F. Espiráculos abdominales, vista lateral G. Espiráculo abdominal H. Proyecciones cónicas IX segmento abdominal, vista ventral. Escalas: A-F: $1 \mathrm{~mm}$; G: 0,2 mm; H: 0,5 mm.

ce de los élitros es de 12,93 $\pm 0,52 \mathrm{~mm}$ en las hembras $(\mathrm{n}=15$; rango $12,12-13,86 \mathrm{~mm})$ y 12,31 $\pm 0,64 \mathrm{~mm}$ en los machos ( $\mathrm{n}=15$; rango 10,82 - 13,27 mm).

La cabeza es redondeada, presenta ojos transversalmente ovales y negros (Fig. 6A). El rostro es sexualmente dimórfico: en las hembras mide 7,19 \pm 0,49 $\mathrm{mm}$ de largo ( $\mathrm{n}=15$; rango $6,51-8,02 \mathrm{~mm}$ ), es curvo y delgado mientras que en los machos es recto, grueso y alcanza una longitud de 5,20 \pm 0,40 $\mathrm{mm}$ ( $\mathrm{n}=15$; rango 4,42 - 5,75 mm) (Fig. 6B). Inserción antenal amplia, visible dorsalmente; en las hembras se localiza más cerca de la base del rostro, en los machos más cerca del ápice. La cabeza y el tercio basal del rostro presentan pocas escamas filiformes, cortas y blancas; cada una se inserta en una perforación pequeña y muy superficial. Los dos tercios distales del rostro son glabros, con perforaciones dispersas y mucho más finas. El escrobo antenal extendido hacia la superficie ventral del rostro.

Antenas geniculadas con clava compacta, de color negro, formadas por once segmentos (Fig. 6C). El escapo es alargado, glabro en la base y con escamas filiformes blanquecinas en el ápice. Los artejos funiculares (segmentos II-VIII) se unen oblicuamente al escapo, presentan setas blancas y muy delgadas. Clava constituida por tres artejos estrechamente unidos (segmentos IX, X y XI); la superficie con pequeñas setas de color marrón claro que le dan un aspecto aterciopelado, especialmente en el último segmento. Mandíbulas esclerotizadas de color negro, con tres dientes. Palpos labiales trisegmentados (Fig. 6D).

Pronoto subtriangular, casi tan largo como ancho. Margen apical curvado, redondeado a los lados; margen basal bisinuado. En las hembras mide 3,22 $\pm 0,12 \mathrm{~mm}$ de largo ( $\mathrm{n}=15$; rango 3,00 - 3,44 mm) y 3,69 $\pm 0,14 \mathrm{~mm}$ de ancho ( $n=15$; rango 3,47 - 3,99 mm); en los machos mide $3,04 \pm 0,22 \mathrm{~mm}$ de largo ( $\mathrm{n}=15$; rango $2,63-3,36 \mathrm{~mm})$ y $3,52 \pm 0,23 \mathrm{~mm}$ de ancho ( $\mathrm{n}=15$; rango 2,99 - 3,88 mm) (Fig. 6E). El pronoto presenta perforaciones pequeñas y poco profundas, la mayoría con una seta corta y blanca en su interior. 


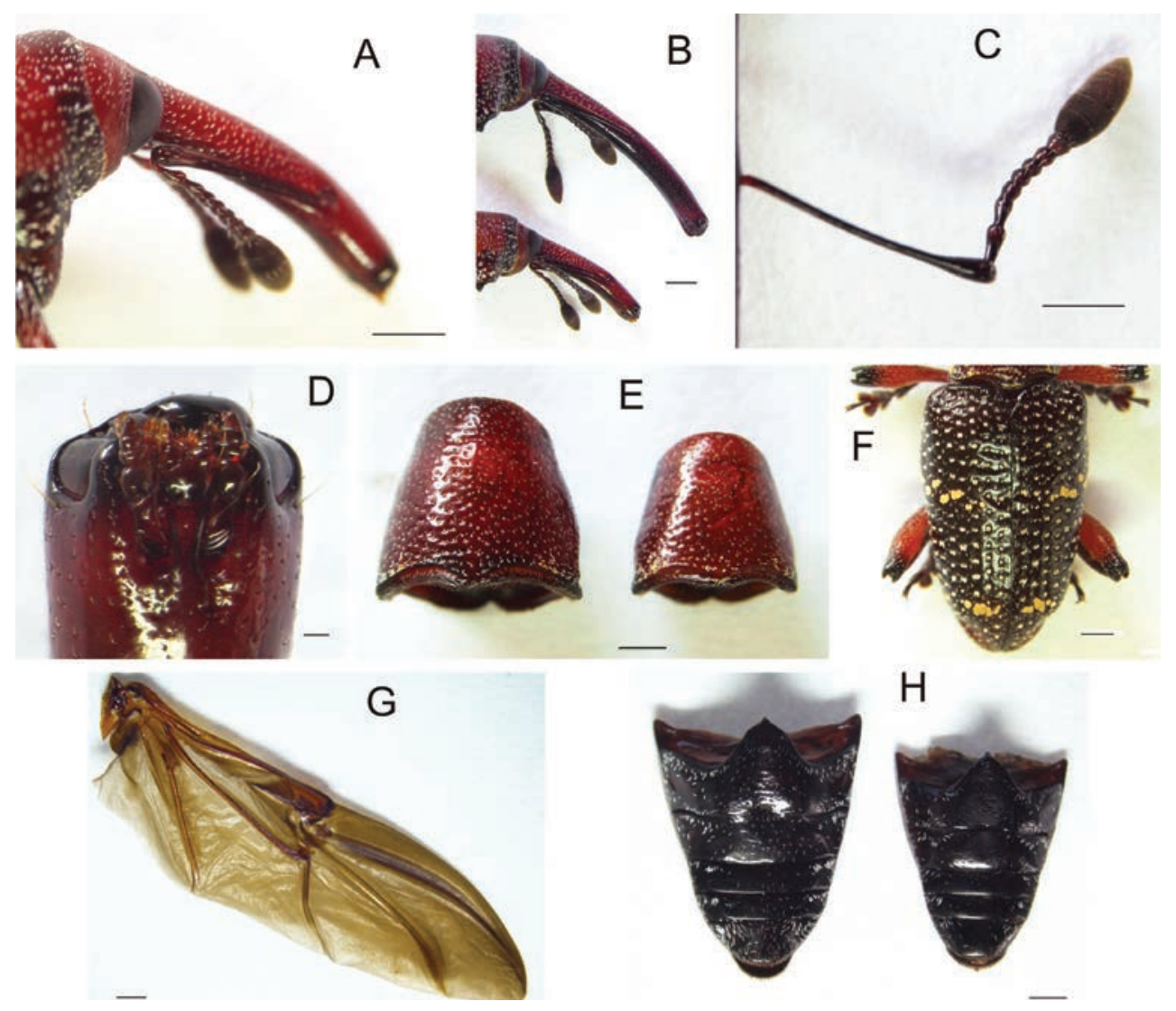

Figura 6. Heilipus lauri, adulto: A. Cabeza, vista lateral macho B. Rostro, vista lateral ( + arriba, đ̧abajo) C. Antena derecha, vista dorsal hembra D. Aparato bucal, vista ventral hembra E. Pronoto, vista dorsal (qizquierda, ổderecha) F. Élitros, vista dorsal macho G. Ala posterior derecha, vista dorsal hembra H. Abdomen, vista ventral (†izquierda, ôderecha). Escalas: A-C: $1 \mathrm{~mm}$; D: 0,1 mm; E-H: 1 mm.

Los élitros tienen forma alargada, son amplios en la base y se estrechan gradualmente mientras cubren los segmentos abdominales; el ápice es redondeado (Fig. 6F). En las hembras alcanzan una longitud de 9,71 $\pm 0,47 \mathrm{~mm}$ $(\mathrm{n}=15$; rango $8,84-10,7 \mathrm{~mm})$ y $5,88 \pm 0,22 \mathrm{~mm}$ en su parte más ancha (n=15; rango 5,50 - 6,24 mm). En los machos miden 9,27 $\pm 0,48 \mathrm{~mm}$ de largo $(\mathrm{n}=15$; rango 8,19 $10,14 \mathrm{~mm})$ y $5,60 \pm 0,34 \mathrm{~mm}$ de ancho ( $\mathrm{n}=15$; rango 4,76 - 6,09 mm). La superficie con perforaciones un poco más grandes y profundas si se comparan con las de la cabeza y el pronoto; cada perforación alberga varias escamas filiformes cortas que semejan pequeños puntos blancos. El carácter diagnóstico de la especie lo constituyen dos manchas transversales en cada élitro: la primera se ubica ligeramente por encima de la línea media, la segunda muy cerca del cayo periapical. Cada mancha está conformada por escamas filiformes, gruesas y de color amarillo opaco, densamente agrupadas en perforaciones grandes, profundas y sin una forma definida.
Las alas posteriores son membranosas y de color marrón oscuro (Fig. 6G). En la región pleural el metepimeron está cubierto por los élitros. El abdomen en vista ventral consta de cinco esternitos de color negro, tanto en machos como en hembras (Fig. 6H). La superficie con escasas escamas filiformes cortas y blancas, la mayoría hacia los bordes de cada esternito.

Patas provistas de escamas alargadas, blancas y delgadas (Fig. 7A). Coxas anteriores contiguas; trocánteres reducidos y triangulares. Los fémures se amplían progresivamente hacia la zona distal, a dos tercios de la base presentan una espina que se extiende hacia el ápice (Fig. 7B); el margen interno de la espina aserrado. Tibias cilíndricas, uncinadas, ligeramente curvas y con un mechón de setas de color marrón claro en el ápice de la superficie ventral; peine tibial presente. Los tarsos divididos en cinco tarsómeros, el tarsómero I alargado; tarsómero III bilobulado y un poco más ancho que el II; tarsómero IV muy pequeño, oculto entre el III; tarsómero V alargado, 

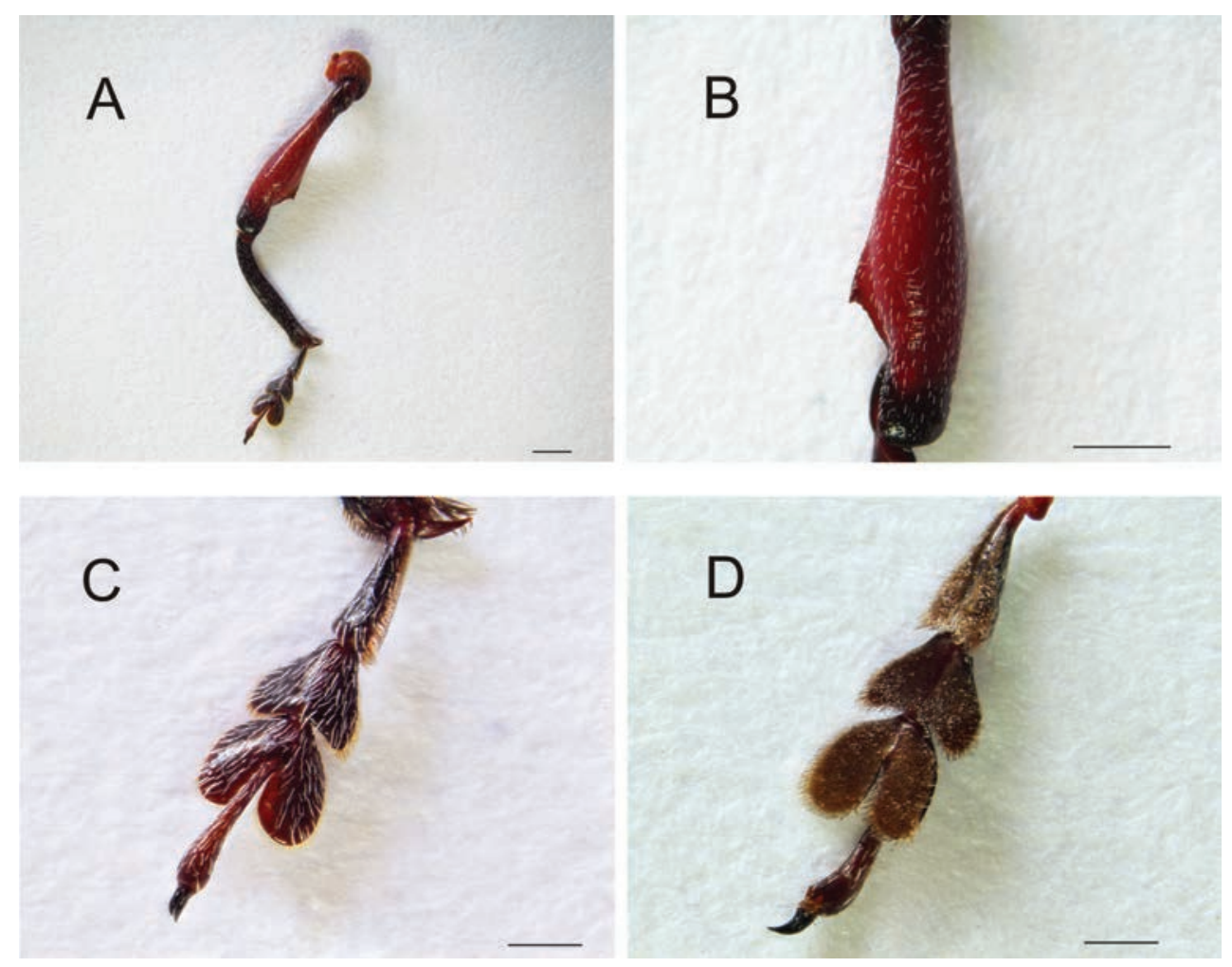

Figura 7. Heilipus lauri, adulto hembra: A. Pata anterior izquierda, vista dorsal B. Fémur anterior derecho, vista dorsal C. Tarso anterior izquierdo, vista dorsal D. Tarso anterior izquierdo, vista ventral. Escalas: A-B: $1 \mathrm{~mm}$; C-D: 0,5 mm.

con dos uñas simples y libres en el extremo distal (Fig. 7C). Los tarsómeros I-III con abundantes setas cortas y de color marrón claro en la cara ventral (Fig. 7D); la cantidad de setas y su proximidad dan a los tarsos un aspecto esponjoso por debajo.

\section{CONCLUSIONES}

Heilipus lauri es una especie espermatófaga, esta condición representa una ventaja para los estados inmaduros del insecto, no solo porque le asegura una fuente de alimentación a la larva sino también porque representa un refugio tanto para la larva como para la pupa, evitando la exposición directa a patógenos, depredadores y parasitoides que puedan afectar su desarrollo. Este mecanismo, basado en la alimentación de semillas y pupación en su interior, sumado a una alta viabilidad del huevo aumenta las posibilidades de supervivencia de la especie. Así, una estrategia exitosa desde el punto de vista biológico cobra importancia económica, debido a que la ausencia de medidas de control puede conducir a altos niveles de daño; lo anterior sin tener en cuenta que la presencia del perforador en las regiones productoras de aguacate ya supone una barrera para el ingreso del producto a mercados especializados.

En síntesis, los resultados reportados en este estudio contribuyen al conocimiento de las poblaciones locales de H. lauri; además, sugieren una estrecha relación biológica morfológica entre poblaciones colombianas y mexicanas de esta especie. Se requieren estudios genéticos que aporten conocimiento sobre el origen de las poblaciones de $H$. lauri presentes en Norte, Centro y Suramérica, que expliquen los factores bióticos y abióticos que han contribuido a su amplia distribución, lo cual la posiciona como una especie exitosa frente a otras de Heilipus sp., asociadas a P. americana.

AGRADECIMIENTOS. A Martha Eugenia Londoño por sus aportes para la realización de este trabajo; a Luisa Fernanda Torres y Luis Carlos Rojas de la Dirección Técnica de Epidemiología y Vigilancia Fitosanitaria del Instituto Colombiano Agropecuario, Seccional Antioquia; a Ovidio Montoya del Centro de Investigación La Selva de Corpoica. Al laboratorio de entomología del Centro de Investigación Palmira de Corpoica por el soporte técnico en microscopía. Al Ministerio de Agri- 
cultura y Desarrollo Rural (MADR) por la financiación del proyecto. A los evaluadores y editores por las sugerencias al manuscrito.

\section{LITERATURA CITADA}

Barber, H.S. (1919). Avocado seed weevils. Proceedings of the Entomological Society of Washington. 21, 53-60.

Boheman, C.H. (1845). In: Genera et species Curculionidum cum sinonimia hujus familiæ. Vol. 8. Part 2. (C.J. Schoenherr).

Caicedo, L., Varón, E., Bacca, T. \& Carabalí, A. (2010). Daños ocasionados por el perforador del aguacate Heilipus lauri Boheman (Coleoptera: Curculionidae) en Tolima (Colombia). Revista Corpoica - Ciencia y Tecnología Agropecuaria, 11, 129-136.

Carabalí, A. (2014). Barrenador grande de la semilla del aguacate. Pp. 229-234. In: Corporación Colombiana de Investigación Agropecuaria - Corpoica (Ed.). Manual técnico actualización tecnológica y buenas prácticas agrícolas (BPA) en el cultivo de aguacate. Medellín, Colombia.

Carabalí, A., Kondo, D.T., Rojas, A., Díaz, A.E., Ramos, P., Quintero, M., Orozco, M.L., Acosta, L.M., Arias, A., Castillo, F., Grijalva, O., Murillas, M. \& Muñoz, J.A. (2009). El grupo MIP, manejo integrado de plagas y enfermedades. Novedades Técnicas, 10, 11-16.

Castañeda, A. (2008). Bioecología del barrenador grande de la semilla del aguacate Heilipus lauri Boheman (Coleoptera: Curculionidae) en la región central de México. Tesis de doctorado. Colegio de Postgraduados, Montecillo, Texcoco, México, 83 p.

Castañeda-Vildózola, A., Equihua-Martínez, A., Franco-Mora, O., González-Huerta, A. \& Palacios-Torres, R.E. (2012). Longevidad, fertilidad y fecundidad de Heilipus lauri Boheman (Coleoptera: Curculionidae: Molytinae) bajo condiciones de laboratorio. Boletín del Museo de Entomología de la Universidad del Valle, 13, 1-7.

Champion, G.C. (1902). Biologia Centrali-Americana. Insecta. Coleoptera. Rhynchophora. Curculionidae. Curculioninae. Vol. IV. Part 4. London, UK.

Costa Lima, A. da. (1956). Insetos do Brasil. Coleópteros, $4^{a}{ }^{a}$ e última parte. Escola Nacional de Agronomia, Brasil, 373 p.

Craw, R.C. (1999). Molytini (Insecta: Coleoptera: Curculionidae: Molytinae). Fauna of New Zealand Number 39. Manaaki Whenua Press. Lincoln, Canterbury, New Zealand, 68 p.

EPPO Global Database. (2016). Heilipus lauri (HEILLA). Distribution. Disponible en: https://gd.eppo.int/taxon/HEILLA/distribution (Consulta: 11 abril 2016).

Gallego, F. L. (1949). Estudios entomológicos. Revista Facultad Nacional de Agronomía Medellín. 10, 121-134.

García, P. (1962). Heilipus lauri Boheman un barrenador de la semilla o hueso del aguacate en México. Tesis de pregrado. Escuela Nacional de Agricultura, Chapingo, México, 107 p.

Hoyos, L.F. \& Giraldo, J. (1984). Reconocimiento de los insectos barrenadores del fruto en el aguacate (Persea americana Mill) y evaluación económica de su daño, en huertos de los departamentos de Caldas y Risaralda. Tesis de pregrado. Universidad de Caldas, Manizales, Colombia, 98 p.

ICA. (2012). Manejo fitosanitario del cultivo del aguacate Hass (Per- sea americana Mill). Medidas para la temporada invernal. Instituto Colombiano Agropecuario ICA. Bogotá D.C., Colombia, 72 p.

ICA. (2014). Distribución de especies cuarentenarias del aguacate Hass en el Oriente de Antioquia y el Norte del Tolima en Colombia. Instituto Colombiano Agropecuario ICA. Bogotá D.C., Colombia, $38 \mathrm{p}$.

ICA. (2015). Boletín epidemiológico. Resultados de vigilancia de especies cuarentenarias de aguacatae var. Hass: Heilipus lauri Boheman, Heilipus trifasciatus Fabricius (Coleoptera: Curculionidae) y Stenoma catenifer Walsingham (Lepidoptera: Oecophoridae) en Cauca Enero-Junio 2015. Disponible en: http://www.ica.gov.co/ Areas/Agricola/Servicios/Epidemiologia-Agricola/Boletines-Epidemiologicos-Agricolas-(1)/2015_DPTO/2015_RVF_BoletinEpidemiologico-VPCO_Cauca-2015-1.aspx (Consulta: 12 abril 2016).

ICA. (2016). Resolución No. 00001507 (22/02/2016). Disponible en: http://www.ica.gov.co/getattachment/81591168-ac45-478a-b84bf054d4e5829b/2016R1507.aspx (Consulta: 11 abril 2016).

Manrique, M.B., Carabalí, A., Kondo, T. \& Bacca, T. (2014). Biología del pasador del fruto Stenoma catenifer Walsingham (Lepidoptera: Elachistidae) y búsqueda de sus posibles enemigos naturales. Boletín Científico Museo de Historia Natural Universidad de Caldas, 18, 79-92.

Marvaldi, A.E. (2003). Key to larvae of the South American subfamilies of weevils (Coleoptera, Curculionoidea). Revista Chilena de Historia Natural, 76, 603-612.

Marvaldi, A.E. \& Morrone, J.J. (1998). Immature stages of Rhyparonotus altarensis (Olliff) (Coleoptera: Curculionidae: Molytinae), with comments on larval characters in Anchonini and Molytinae. Journal of the New York Entomological Society, 106, 95-104.

Marvaldi, A.E. \& Lanteri, A.A. (2005). Key to higher taxa of South American weevils based on adult characters (Coleoptera, Curculionoidea). Revista Chilena de Historia Natural, 78, 65-87.

May, B.M. (1981). Immature stages of Curculionoidea - the weevils of The Snares islands, New Zealand. New Zealand Journal of Zoology, 8, 255-280.

May, B.M. (1993). Larvae of Curculionoidea (Insecta: Coleoptera): a systematic overview. Fauna of New Zealand Number 28. Manaaki Whenua Press. Lincoln, Canterbury, New Zealand, 226 p.

Medina-Quiroz, F., López-Martínez, V., Alia-Tejacal, I., GarcíaRamírez, M.J., Guillén-Sánchez, D., Andrade-Rodríguez, M., Villegas-Torres, O.G. \& Acosta-Durán, C.M. 2010. Barrenador grande del hueso del aguacate (Heilipus lauri Boheman) en Tepoztlán, Morelos. Investigación Agropecuaria, 2, 67-75.

Nylin, S. \& Gotthard, K. (1998). Plasticity in life-history traits. Annual Review of Entomology, 43, 63-83.

Palacios-Torres, R.E., Castañeda-Vildózola, A., Sánchez-Pale, J.R., Franco-Mora, O. \& Vargas-Rojas, L. (2016). Interaction of two avocado fruit insects in the same agroecological area in Mexico. Pan-Pacific Entomologist. 92, 100-103.

Peña, J.E. (Ed.). (2013). Potential Invasive Pests of Agricultural Crops. CAB International, London, U.K.

Peterson, E.B. \& Orden, D. (2008). Avocado pests and avocado trade. American Journal of Agricultural Economics, 90, 321-335.

Ramírez, J.G., Castañeda, D.A. \& Morales, J.G. (2014). Estudios etiológicos de la marchitez del aguacate en Antioquia-Colombia. Revista Ceres, 61, 050-061. 
Rubio, J.D., Posada, F.J., Osorio, O.I., Vallejo, L.F. \& López, J.C. (2009). Primer registro de Heilipus elegans Guérin-Méneville (Coleoptera: Curculionidae) atacando el tallo de árboles de aguacate en Colombia. Revista U.D.C.A Actualidad y Divulgación Científica, 12, 59-68.

Salgado, M.L. \& Bautista, N. (1993). El barrenador grande del hueso del aguacate (Heilipus lauri Boheman) en Ixtapan de la Sal, México. Pp. 225-229. In: Fundación Salvador Sánchez Colín CICTAMEX, S.C. (Ed.). Memoria 1993. Coatepec Harinas, México.

Sandoval, A., Forero, F., García, J. \& Bonilla, M. (2014). Cosecha, manejo poscosecha y agroindustria. Pp. 314-357. In: Corporación Colombiana de Investigación Agropecuaria - Corpoica (Ed.). Manual técnico actualización tecnológica y buenas prácticas agrícolas (BPA) en el cultivo de aguacate. Medellín, Colombia.

Silva, L.K.F., Arthur, V., Nava, D.E. \& Parra, J.R.P. (2006). Tratamento quarentenário em ovos de Stenoma catenifer Walsingham (Lepidoptera: Elachistidae), com radiação gama do Cobalto-60. Boletín de sanidad vegetal. Plagas, 32, 507-512.

Sousa, W.O. de., Santos, G.B. \& Rosado-Neto, G.H. (2004). As- pectos morfológicos de Pseudopiazurus obesus (Boheman) e considerações sobre a sinonímia com Pseudopiazurus papayanus (Marshall) (Coleoptera: Curculionidae). Acta Biológica Paranaense, 33, 167-179.

Stearns, S.C. (1992). The Evolution of Life Histories. Oxford University Press, Oxford.

Vallejo, L.F., Sánchez, R. \& Salgado, M. (2007). Redescripción del adulto y descripción de los estados inmaduros de Cosmopolites sordidus Germar, 1824 (Coleoptera: Curculionidae), el picudo negro barrenador del plátano en Colombia. Boletín Científico Museo de Historia Natural, 11, 361-375.

Vanin, S.A. \& Gaiger, F. (2005). A new spermophagous species of Heilipus Germar from the Amazonian Region (Coleoptera, Curculionidae, Molytinae). Revista Brasileira de Entomologia, 49, 240-244.

Wibmer, G.J. \& O'Brien, C.W. (1986). Annotated checklist of the weevils (Curculionidae sensu lato) of South America (Coleoptera: Curculionoidea). Memoirs of the American Entomological Institute. 39, 1-563. 